\title{
Extract Tacit Knowledge in the Learner Model of the Smart Tutoring System
}

\author{
https://doi.org/10.3991/ijet.v15i04.11781 \\ Fatima-Zohra Hibbi $\left.{ }^{\square}\right)$, Otman Abdoun, El Khatir Haimoudi \\ Abdelmalek Essaâdi University, Larache, Morocco \\ fatima.zohra.official@gmail.com
}

\begin{abstract}
Knowledge management (KM) is one of the main factors that have become extremely popular in recent years. KM is the processes which people explain information data using scientific and technological media and summarize it into concepts and rules to generate knowledge. This later can be implicit or explicit one. The aim of this contribution is to convert the tacit knowledge into explicit using Metaheuristics techniques. This paper aims to develop a model for converting tacit knowledge into explicit knowledge, using the Metaheuristics algorithm for the E-learning platform. For that purpose, the knowledge conversion process will respect the following steps: define the source of tacit knowledge and their methods, classify the tacit knowledge, then we evaluate the implicit knowledge conversion.
\end{abstract}

Keywords-Knowledge Management, Metaheuristics, Tacit Knowledge, Competitive learning Algorithm

\section{$1 \quad$ Introduction}

The integration of knowledge management and E-learning technology knows nowadays an interesting from the researches. This integration provides an organization and delivers traditional courses. Schmidt proposes a solution to encourage learning in institution. He explains that the isolation of learners returns to the luck of their attention to the context in learning process [1]. Walk's and Agarwal's propose a model that is based on Nonaka and Takenuchi model of conversion tacit into explicit knowledge using four steps: socialization, externalization, combination and internalization [2].

Nevertheless, to enhance this integration; the uses of a Smart Tutoring System (STS) is needed. The STS provides feedback on the actions of learners without the intervention of human being. This system contains a learner model wish has the general information of the learner, their knowledge and their level.

This information is the traces of learner collected from the E-learning platform. However, the problem is the difficulty of the extraction of the tacit knowledge in the student model. The aim of this paper is to convert tacit knowledge into explicit knowledge in the learner model of the smart tutoring system.

The article is presented as follows: after presenting the problems of the student model in Smart Tutoring System and the solution proposed, we present the metaheu- 
ristics methods and the choice of Competitive learning algorithm (CLA) as a best metaheuristics technique. In the fourth section, we describe our proposed approach: integration of metaheuristics to convert tacit knowledge into explicit one in student model of Smart tutoring System. Finally, this article will be ended with a conclusion and future works.

\section{Student Model}

The student model contains all the information about the learner in E-Learning platform. It includes the general information and personal one; such as their knowledge, behaviors and their learning level.

The intelligent tutoring systems (STS) that we are proposed (see figure1) include a student model. This later contains a lot of information, among these information we are interested in knowledge acquisition of learner during an E-learning process. The knowledge is divided in two parts implicit and explicit knowledge. The first one describe their understanding level for a pedagogical sequence, the system can collect the learner development process and their assessments. Nevertheless, the implicit knowledge is embedded in the mind and difficult to share.

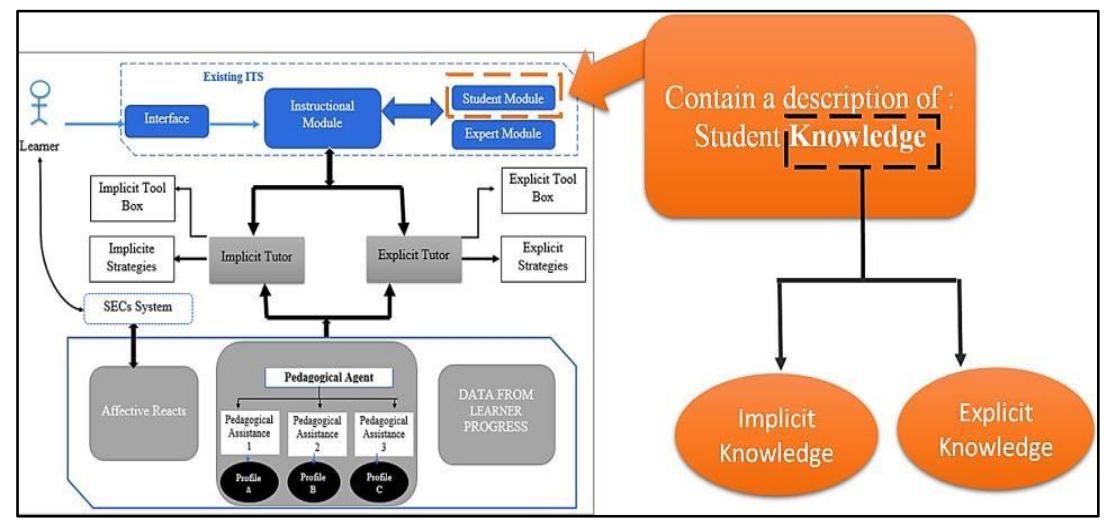

Fig. 1. The student model in smart tutoring system

\section{Metaheuristics Techniques}

The term metaheuristics is a new paradigm of searching algorithms and solve complex optimization problems. This concept was proposed to identify a high level heuristics to lead other heuristics for a more efficient evolution in the research space.

The metaheuristics algorithm is very beneficial due to their ability to explore an efficient space regardless of size and space.

Optimization algorithms are generally devised on two parts deterministic and stochastic. The first one produces the same results. The second one wish we are interested in have a specific and good result since they can easily escape from local minima 
even with their low convergence speed. The figure bellow (Fig.2) summary the principal optimization algorithms

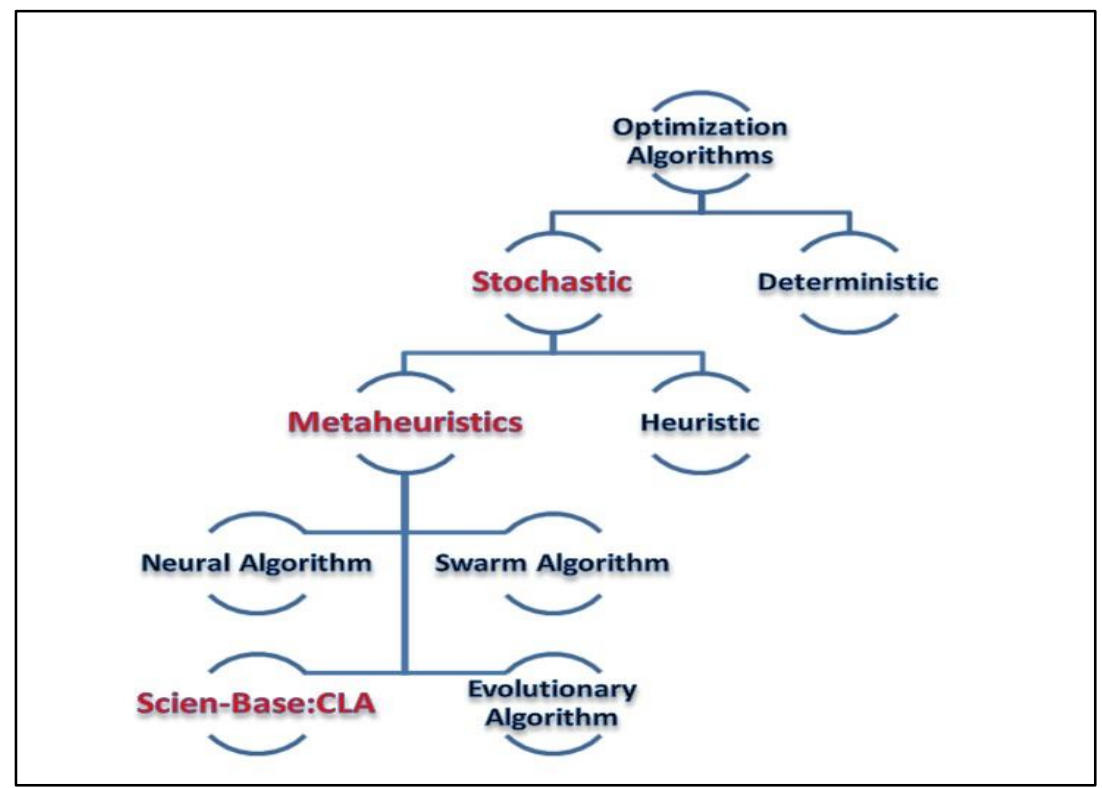

Fig. 2. The stochastic optimization algorithms

In this paper, to solve the problem of extraction of tacit knowledge we will use the competitive learning Algorithm (CLA) [3]. This later is based on the ICA (independent component analysis) and TLBO (Teaching learning based optimization) concepts. In this algorithm, learners are distributed in a various classes randomly, and then they can improve their knowledge using tree diverse learning methods: competitive, personal and teacher-oriented learning [4-5].

The choice of CLA algorithm is not randomly; the CLA shows a very good result in unimodal and multimodal functions. This algorithm provides very competitive results and outperforms ICA and TLBO on the most of multimodal functions. The CLA able to enhance highly competitive results compare to PSO and GA algorithms. The figure 3 shows the efficient result of CLA algorithm.

\begin{tabular}{|c|c|c|c|c|c|c|}
\hline & CLA & ICA & TLBO & GWO & PSO & GA \\
\hline \multicolumn{7}{|l|}{ Sphere } \\
\hline Ave & $6.3559 \mathrm{e}-04$ & $5.3248 \mathrm{e}+03$ & 723.1606 & $1.6390 \mathrm{e}+03$ & 959.5678 & 831.0194 \\
\hline Std & 0.0014 & $8.9021 \mathrm{e}+03$ & $3.2265 \mathrm{e}+03$ & $6.9679 \mathrm{e}+03$ & $4.5618 \mathrm{e}+03$ & $3.6531 \mathrm{e}+03$ \\
\hline BestSolution & $5.2350 \mathrm{e}-27$ & 22.8169 & $5.1291 \mathrm{e}-14$ & $5.9584 \mathrm{e}-08$ & 0.80322 & 14.3749 \\
\hline Time & 5.0421 & 5.1271 & 4.9632 & 8.0441 & 4.8383 & 6.2785 \\
\hline Population & 200 & 200 & 200 & 200 & 200 & 200 \\
\hline
\end{tabular}

Fig. 3. Efficient result of CLA Algorithm 


\section{The Proposed approach: Convert Tacit Knowledge Into Explicit Knowledge}

In this section, we describe our proposed approach on how Competitive Learning Algorithm and Neural Network can be used to convert tacit knowledge to explicit knowledge. At the beginning, the source of the tacit knowledge is checked from a list of characteristics, and then we collect the implicit knowledge using such techniques. This later is described in the process (Figure 4). The data collected will be classified using metaheuristics techniques.

The design of our proposed approach and its components is illustrated in figure 4. Our approach consists of four stages: the sources of tacit knowledge, the techniques of the implicit knowledge, the classification and the adaptation (recommendation).

Each stage in our process is described below:

Step1: The source of the tacit knowledge (T.K):

The first step is define the source of the tacit knowledge: During the tasks assigned to a group of $\mathrm{P}=\{\mathrm{P} 1, \ldots, \mathrm{Pn}\}$. Each student will be given a set of characteristics $\mathrm{C}=$

$\{\mathrm{C} 1, \ldots, \mathrm{Cn}\}$. The forms of the tacit knowledge are listed as bellow:

- C1: Insight

- C2: Intuition

- C3: Common Knowledge

- C4: Inherent talent

- C5: Skills experiences

After this, a training data is created; it contains the whole of the characteristics (source of T.K) and then learning Dataset will be presented to the Competitive Learning Algorithm to optimize the space of the source of tacit knowledge. Afterward, we passed to the step 2 which is described below:

Step2: The techniques of the tacit knowledge (T.K):

The second step is to define the method to obtain tacit knowledge: there are a several techniques to collect the tacit knowledge such as:

- Record the activities of the learners;

- Preparation of Knowledge Acquisition form;

- Create a space in the platform where learners can note down their observation;

- Etc.

In this step, the system collects the data from the pedagogical resource listed above in the E-learning platform and then we move on to the second internship:

Step3: The Classification of the Data:

The step 3 is concerned to classify the collected tacit knowledge: this step is very important it includes two stages; the first one is to collect the data from the steps 1 and 2; after that we will apply the neural network method to classify the result obtained from the twice stages.

Step4: The Adaptation (Recommendation) 
After identifying the source and the method of tacit knowledge and classifying the data, we aim to provide a relevant learner model. This model contains the explicit knowledge and the converting implicit to explicit knowledge through metaheuristics techniques.

Concisely, the adaptation of the learner model can be done as follow:

- Identifying the most tacit knowledge used by the learner

- Create learners cluster based on the classification results.

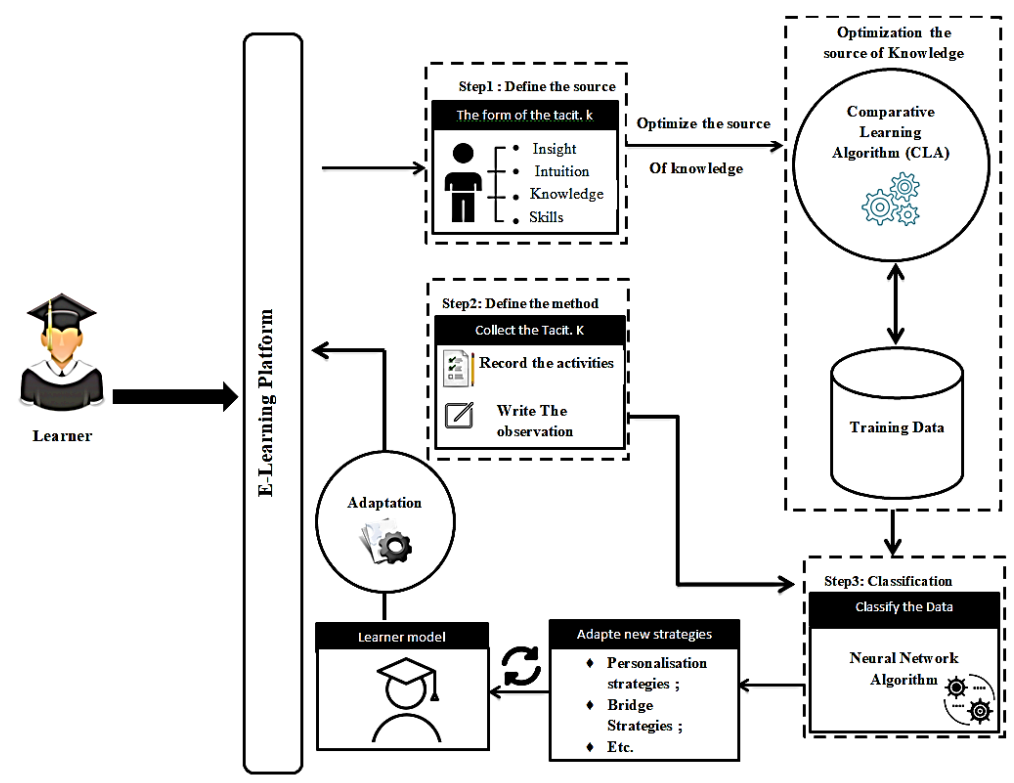

Fig. 4. The process of converting tacit knowledge to explicit knowledge.

\section{Conclusion and Future Work}

This paper presents the problem of tacit knowledge in a student model in a smart tutoring system, analyzing the diverse metaheuristics techniques, deduct the uses of the competitive learning algorithm as a best metaheuristic algorithm, and we propose a new convert model of tacit knowledge to explicit one.

We are positive that this approach will make learner model benefits from the advantages of using both: implicit and explicit knowledge to improve learning process.

Concrete implementation of these general suggestions and validations of their effectiveness are however left as future works. These experimentations will be done on the E-learning Platform of Polydisciplinary Faculty in Larache, part of Abdelmalek Essaadi University in Morocco. 


\section{$6 \quad$ References}

[1] J. JUDRUPS, (2014). Analysis of Knowledge Management and E-Learning Integration Models. ICTE in Regional Development, Valmiera, Latvia.

[2] C E. BECK, G R. SCHORNACK, (2005). A Systems Model for Knowledge Management: A Rhetorical Heuristic Process. Proceedings of the 38th Hawaii International Conference on System Sciences. https://doi.org/10.1109/hicss.2005.55

[3] THOMAS C. REEVES and all (2002), Usability and Instructional Design Heuristics for ELearning Evaluation, World Conference on Educational Multimedia, Hypermedia and Telecommunications.

[4] Z CHUNHUA, (2008). E-learning: The New Approach for Knowledge Management (KM), Inter- national Conference on Computer Science and Software Engineering.

[5] J. AL-SADI, B. ABU-SHAWAR, AND T. SARIE, (2008). The Relationship between Knowledge Management and e-Learning, Proceedings of the 2008 International Conference on E- Learning, E-Business, Enterprise Information Systems, and E-Government, IEEE.

\section{Authors}

Fatima-Zohra Hibbi is PHD Student in Abdelmalek Essaadi University, Larache, Morocco. (E-mail: Fatima.zohra.official@gmail.com)

Otman Abdoun is a professor in department of computer science at Pluridisciplinary Faculty Larache in Abdelmalek Essaadi University, Morocco. (E-mail: abdoun.ot man@gmail.com)

El Khatir Haimoudi is a professor in department of computer science in Abdelmalek Essaadi University, Larache, Morocco. (E-mail: elkhatir@gmail.com)

Article submitted 2019-09-28. Resubmitted 2019-11-26. Final acceptance 2019-11-27. Final version published as submitted by the authors. 



iJET - Vol. 15, No. 4, 2020

\section{Imprint}

iJET - International Journal of Emerging Technologies in Learning

http://www.i-jet.org

\section{Editor-in-Chief}

Dominik May, University of Georgia, United States

\section{Executive Editor}

Michael E. Auer, CTI Frankfurt/Main, Vienna, New York, Bengaluru

\section{Associate Editors-in-Chief}

Martin Ebner, TU Graz, Austria

Mohammad Khalil, University of Bergen, Norway

\section{Associate Editors}

Neelakshi Chandrasena Premawardhena, University of Kelaniya, Sri Lanka

Christina Merl, Vienna, Austria

Jalal Nouri, Stockholm University, Sweden

Stamatis Papadakis, University of Crete, Greece

Valerie Varney, RWTH Aachen University, Germany

\section{Technical Editor}

Sebastian Schreiter, Lagorce, France

\section{Editorial Board}

A. Y. Al-Zoubi, Princess Sumaya University for Technology Amman, Jordan Gerald Friedland, International Computer Science Institute, Berkeley (CA), USA

Andreas Holzinger, Medical University Graz, Austria

Dr. George S. Ioannidis, University of Patras, Greece

Andreja Istenic Starcic, Slovenia University of North Texas, US

Barbara Kerr, Concordia University Montreal, Canada

Lars Knipping, TU Berlin, Germany

Massimo Marchiori, World Wide Web Consortium (W3C) at MIT

Andreas Pester, Carinthia University of Applied Sciences, Austria

Rob Reilly, University of Memphis and MIT Media Lab, USA

Cornel Samoila, Transilvania University Brasov, Romania

Mario Žagar, University of Zagreb, Croatia

\section{Indexing}

International Journal of Emerging Technologies in Learning is indexed in Clarivate Analytics ESCl, Elsevier Scopus, El Compendex, DBLP, Ulrich, EBSCO, INSPEC, LearnTechLib, and Google Scholar.

\section{Publication Frequency}

Bi-Monthly

\section{ISSN}

1863-0383

\section{Publisher}

International Association of Online Engineering (IAOE)

Kirchengasse 10/200

A-1070 Vienna

Austria 